\title{
Modulators Using DPS-DNG-Layered Structure
}

\author{
J. H. Shahbazian \\ Department of Electrical and Computer Engineering, Tufts University, Medford, MA 02155, USA \\ Correspondence should be addressed to J. H. Shahbazian, jshahbazian@msn.com
}

Received 16 November 2011; Accepted 8 December 2011

Academic Editor: K. Y. Song

Copyright () 2012 J. H. Shahbazian. This is an open access article distributed under the Creative Commons Attribution License, which permits unrestricted use, distribution, and reproduction in any medium, provided the original work is properly cited.

The influence of an applied electric field on a periodic structure composed of alternating layers of isotropic DPS (double positive index of refraction) and anisotropic DNG (double negative index of refraction) has been studied and shown that in the presence of an external electric field, one can convert the polarization state of the incident electromagnetic wave into a desired polarization state, change the phase and amplitude, and induce birefringence. Here, theoretical and numerical results of investigation of phase and amplitude modulator in the presence of an applied electric field are presented.

\section{Introduction}

In 1968, Veselago [1] postulated and studied theoretically the plane wave propagation in a medium called left handed material (LHM) or double index of refraction (DNG) material. These class materials play important role in science and technology for their broad usage in industry. The DNG materials are artificial structures with unusual electromagnetic properties compared with the conventional materials.

For these materials, the electromagnetic wave propagates antiparallel to the Poynting vector and the angle of refraction is negative, which means electromagnetic waves at the interface of positive refractive index $(n)$ and negative refractive index materials would refract on the opposite side of the normal to the interface compared to ordinary materials. When these materials are integrated in a periodic structure, they would act as photonic band-gap (PBG) materials, and in the result the structure is able to manipulate the electromagnetic wave propagation. For these structures, there is/are a range of frequencies at which the light propagation is strictly forbidden [2-8]. One can change the distribution of charges and reshape the lattice of the material by applying an electric field. The electromagnetic wave impinged on the material in the presence of an electric field will experience different refractive indices (index ellipsoid) and travel with different speeds [9] and change the polarization. In this paper, theoretically and numerically the changes of the polarization state of the incident beam using the DPS-DNG structure as an of amplitude and phase modulator, in the presence of an applied electric field, are studied.

\section{Theory and Results}

As shown in [9] and here, also, to find the refractive indices of DPS-DNG heterostructure, the index ellipsoid is used. The impermeability tensor, which depends on the distribution of the charges [9-12], can be defined as $\eta_{i j}=\varepsilon_{0} \mu_{0} /(\varepsilon \mu)_{i j}$ with $i, j=1,2,3$ where $\varepsilon_{0}$ and $\mu_{0}$ are electric permittivity and magnetic permeability of the vacuum and $\varepsilon_{i j}$ and $\mu_{i j}$ are electric permittivity and magnetic permeability of the DNGDPS layers. Applying an electric field would redistribute the charges in the structure and would change the impermittivity tensor as discussed in [9] in the form of $\eta_{i j}(E)-\eta_{i j}(E=0)=$ $\sum_{k} r_{i j k} E_{k}$, where $r_{i j k}$ is the electro-optical tensor. Writing the wave equation $\nabla \times(\nabla \times \mathbf{E})+\varepsilon \mu\left(\partial^{2} / \partial t^{2}\right) \mathbf{E}=0$ and using the relation of the electric field and the displacement field vector $\mathbf{D}$ and taking the electric field in $+z$ direction as shown in Figure 1, the equation of motion equivalent to the wave equation in an anisotropic medium in the presence of an electric field can be written as

$$
\left[\frac{\partial}{\partial z}+\frac{1}{c} N \frac{\partial}{\partial t}\right] \mathbf{D}=0
$$




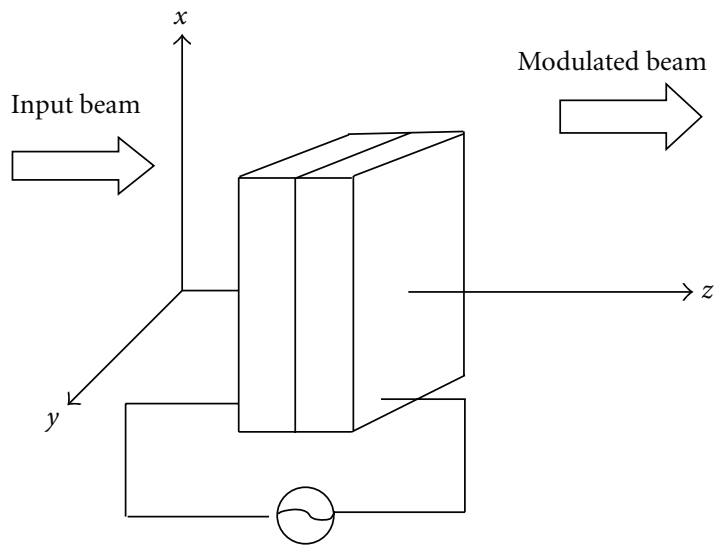

FIgURE 1: Geometrical structure of the heterostructure.

where $z$ is the direction of propagation, $c$ is the speed of light, $\mathbf{D}$ is the displacement field vector in the form:

$$
\begin{aligned}
D(z, t)= & A_{1}(z, t) \exp \left[i\left(\omega t-k_{1} z\right)\right] \mathbf{e}_{1} \\
& +A_{2}(z, t) \exp \left[i\left(\omega t-k_{2} z\right)\right] \mathbf{e}_{2} .
\end{aligned}
$$

$e_{i}$ are the polarization vectors of the normal modes, $A_{i}$ are the normal mode amplitudes, $k_{i}=\omega n_{i} / c$, and $N$ is the refractive index and a $2 \times 2$ matrix shown in [9]:

$$
N=\left(\begin{array}{cc}
n_{1} & -\frac{n_{1}^{2} n_{2}^{2}}{n_{1}+n_{2}} \Delta \eta_{12} \\
-\frac{n_{1}^{2} n_{2}^{2}}{n_{1}+n_{2}} \Delta \eta_{21} & n_{2}
\end{array}\right) .
$$

Here, $\Delta \eta_{i j}$ is perturbation due to the electric field. Substituting the electric displacement vector from (2) in the equation of motion (1) yields:

$$
\begin{aligned}
\left(\frac{\partial}{\partial z}\right. & \left.+\frac{n_{1}}{c} \frac{\partial}{\partial t}\right) A_{1}+i A_{1} \frac{\omega}{c} \Delta N_{11} \\
& =-i A_{2} \frac{\omega}{c} \Delta N_{12} \exp \left[i\left(k_{1}-k_{2}\right) z\right], \\
\left(\frac{\partial}{\partial z}\right. & \left.+\frac{n_{2}}{c} \frac{\partial}{\partial t}\right) A_{2}+i A_{2} \frac{\omega}{c} \Delta N_{22} \\
& =-i A_{2} \frac{\omega}{c} \Delta N_{12} \exp \left[i\left(k_{1}-k_{2}\right) z\right] .
\end{aligned}
$$

One can write

$$
\Delta N_{i j}=\left(\begin{array}{cc}
-\frac{1}{2} n_{1}^{3} \Delta \eta_{11} & -\frac{n_{1}^{2} n_{2}^{2}}{n_{1}+n_{2}} \Delta \eta_{12} \\
-\frac{n_{1}^{2} n_{2}^{2}}{n_{1}+n_{2}} \Delta \eta_{21} & -\frac{1}{2} n_{2}^{3} \Delta \eta_{22}
\end{array}\right),
$$

where $i, j=1,2$ correspond to the layers of the structure. Equation (4) are the mode amplitude equations.

The structure used here contains alternating layers of DPS and DNG. For the DNG layer, SiC nanospheres embedded in the $\mathrm{MgB}_{2}[2,9,13]$ are used. For the DPS layer both electric permittivity and magnetic permeability are constant and positive and for DNG layer, electric permittivity and magnetic permeability are functions of frequency and are given by

$$
\begin{aligned}
& \varepsilon_{y y}(\omega)=1+\frac{4.8412^{2}}{2.1101^{2}-\omega^{2}}, \\
& \mu_{y y}(\omega)=1+\frac{4.2295^{2}}{3.6698^{2}-\omega^{2}},
\end{aligned}
$$

where $\omega=2 \pi f$ and $f$ is the frequency in THz.

If light propagates in the $z$ direction, the equation of the index ellipsoid for this layered structure can be written as

$$
x^{2}\left(\frac{1}{n_{o}^{2}}+r_{13} E_{z}\right)+y^{2}\left(\frac{1}{n_{o}^{2}}+r_{13} E_{z}\right)+z^{2}\left(\frac{1}{n_{e}^{2}}+r_{33} E_{z}\right)=1,
$$

where for $r_{i j k}$, the electro-optic coefficient, the abbreviated notation has been used, $n_{x}=n_{y}=n_{o}-n_{0}^{3} r_{13} E_{z} / 2$ and $n_{z}=$ $n_{e}-n_{e}^{3} r_{33} E_{z} / 2, n_{o}$ is ordinary index of refraction, and $n_{e}$ is the extraordinary index of refraction [2]. The birefringence will be

$$
n_{z}-n_{y}=\left(n_{e}-n_{o}\right)-\frac{1}{2} E_{z}\left(n_{e}^{3} r_{33}-n_{o}^{3} r_{13}\right) .
$$

Using an applied voltage, $V=E d$, where $d$ is the thickness of the unit cell. The retardation can be defined as

$$
\begin{aligned}
\Gamma & =\frac{\omega}{c} d\left(n_{z}-n_{y}\right)=\frac{\omega}{c} d\left(n_{e}-n_{o}\right)-\frac{\omega}{c} V\left(n_{e}^{3} r_{33}-n_{o}^{3} r_{13}\right) \\
& =\frac{2 \pi}{\lambda} d\left(n_{e}-n_{o}\right)-\frac{2 \pi}{\lambda} V\left(n_{e}^{3} r_{33}-n_{o}^{3} r_{13}\right) .
\end{aligned}
$$

2.1. Amplitude Modulation. Applying electric field will induce birefringence. If the incidence beam at input lets say has TE polarization at output, the polarization could change to TM polarization and in the result, the energy flow can be controlled. This happens if in (5), $\Delta \eta_{i j} \neq 0$ for $i \neq j$ or $\Delta \eta_{i j}=$ 0 for $i=j$. Due to the applied electric fields, the modes are coupled and there is pure amplitude modulation. Taking the applied electric field as a traveling field $E=E_{m} \sin \left(\omega_{m} t-k_{m} z\right)$ and $n_{1} \simeq n_{2}=n$, and using the boundary conditions at the input $A_{1}(0, t)=A_{0}$ and $A_{2}(0, t)=0$, the solutions to (4) at the output $z=d$ can be written as

$$
\begin{aligned}
& A_{1}(d, t)=A_{0} \cos [\delta \sin (\omega t-\varphi)], \\
& A_{2}(d, t)=i A_{0} \cos [\delta \sin (\omega t-\varphi)],
\end{aligned}
$$

where the modulation index is; $\delta=\kappa d \sin \theta / \theta, \kappa=$ $\left(\omega n_{o}^{3} / 2 c\right) r_{13 \gamma} E_{\gamma}$ and $\theta=\omega / 2 c\left(n_{o}-n_{m}\right) d$, the out put phase is, $\varphi=\left(\omega_{m} / 2 c\right)\left(n+n_{m}\right) d, n_{o}$ is the ordinary index of refraction and $d$ is the thickness of DPS-DNG layers, $d=d_{1}+d_{2}, d_{1}$ is the thickness of the DPS, which is about $135 \mathrm{~nm}$, and $d_{2}$ is the thickness of the DNG layer and is about $150 \mathrm{~nm}$.

The half-wave voltage is the voltage that the polarization of the incidence wave changes from TE to TM or vice versa. This is the voltage that retardation becomes $\Gamma=\pi$ :

$$
V_{\pi}=\frac{1}{\left(n_{e}^{3} r_{33}-n_{o}^{3} r_{13}\right)}\left[\frac{\lambda}{2}-d\left(n_{e}-n_{o}\right)\right] .
$$




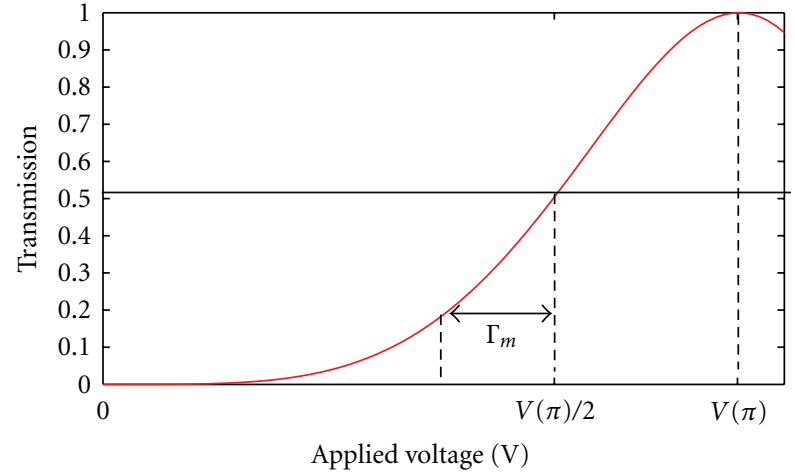

Figure 2: Transmission versus applied electric field.

If the modulation is biased by retardation of $\pi / 2$, which can be achieved by applying the proper electric filed, or by placing a quarter wave plate before the modulator, the modulation voltage $\left(V_{m}\right)$ will cause the transmitted wave to remain in the same shape (amplitude, phase) as the incidence wave, and the retardation will be $\Gamma=\Gamma_{m} \sin \omega_{m} t+\pi / 2$ where $\Gamma_{m}$ is modulator retardation and $\Gamma_{m}=\pi\left(V_{m} / V_{\pi}\right)$. If $\Gamma_{m} \ll 1$, the output wave is a duplication of the input wave; $I_{0} \approx$ $I_{i} \Gamma_{m} \sin \omega_{m} t$ and if $\Gamma_{m} \gg 1$, the output wave is distorted and higher order of harmonics will appear.

Using Jones Calculus, the transmission can be written as

$$
T=\sin ^{2} \frac{\Gamma}{2} .
$$

In Figure 2, the transmission versus applied electric field is shown. The maximum transmission is when $V=V_{\pi}$. In this figure, where the variation of transmission with applied voltage is linear, the modulator is biased which causes a 50\% transmission $[12,14,15]$.

2.2. Phase Modulation. In (5), if $\Delta \eta_{i j}=0$ for $i \neq j$, the modes become uncoupled. In this case, polarization of the traveling waves would not change, but the output phase will change and the solutions to the equation of motion become

$$
A_{i}(d, t)=A_{0 i} \exp [i \delta \sin (\omega t-\varphi)] .
$$

This is the phase modulation case. In this case, the applied electric field does not change the state of polarization (TE or TM), but changes the phase of the output waves by the modulation index $\delta$.

In Figure 3, the modulation index versus the length of the layerd structure, $d=2 \pi c / \omega\left(n-n_{m}\right)$, is shown. The maximum modulation appears when $(\omega / 2 c)\left(n_{o}-n_{m}\right) d=\pi / 2$ and the modulation index becomes $\delta_{\max }=\left(\omega / \omega_{m}\right)\left(n^{3} /\left(n_{o}-\right.\right.$ $\left.n_{m}\right) r_{13 \gamma} E_{m \gamma}$, where $E_{m \gamma}$ is $\gamma$ component of modulation electric field.

In $[2,13]$, the unusual photonic gaps, called non-Bragg gaps, have been studied and emphasized for TE- and TMpolarized incidence waves, in [9] the non-Bragg band gaps for TE polarized incidence waves have been emphasized on and shown in the presence of an electric field. The pass/forbidden bands can be manipulated. In Figure 4, the transmittances band (pass band) for electromagnetic waves on

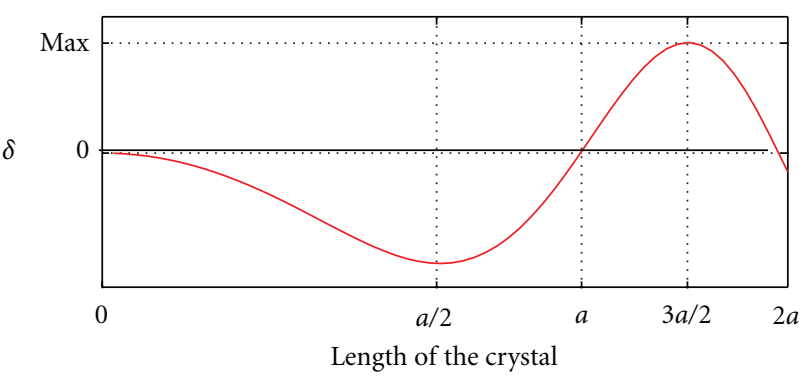

Figure 3: Modulation index versus the lenghth of the layerd structure.

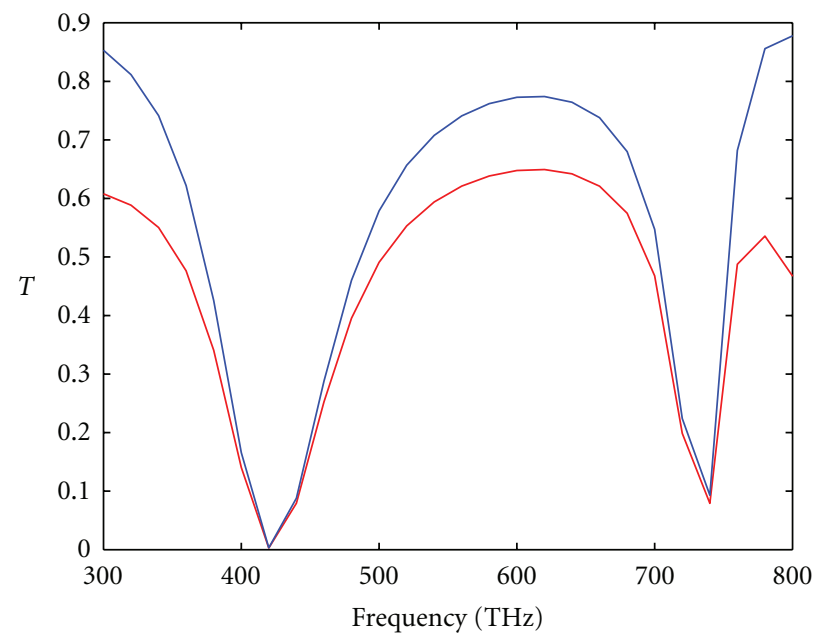

FIGURE 4: The reflectance of the visible electromagnetic wave on DPS-DNG structure in presense of external electric field.

isotropic DPS and nonisotropic DNG layers are shown. The blue line represents the transmittance in the presence of up to $10^{6} \mathrm{~V} / \mathrm{cm}$ applied electric field and the red line shows the transmittance in the prsence of a $10^{8} \mathrm{~V} / \mathrm{cm}$ electric field; but with using the result developed in this manuscript, one can generalize the reflectance/transmittance of electromagnetic waves on this periodic structure for both TE and TM polarizations without worrying about the polarization.

\section{Conclusion}

Applying an electric field which varies sinusoidally in time will cause the propagating wave to follow the electric field, this will not only widen the forbidden/pass bands but also will cause phase or amplitude modulation and change the polarization state of the wave and/or phase of the out going waves. With using the result of this manuscript, the polarization of the incidence wave is not of big concern, since one can change the polarization, from TE to TM or vice versa, with applying electric field. Experimentally, a tunable laser could be used as a variable frequency source at normal incidence to illuminate the DPS-DNG layered structure. 


\section{References}

[1] V. G. Veselago, "The electrodunamics of substances with simultaneously negative values of $\varepsilon$ and $\mu$," Soviet Physics Uspekhi, vol. 10, pp. 509-514, 1968.

[2] J. H. Shahbazian and A. S. Karakashian, "Reflection of visible light on the anisotropic slab of DPS-DNG layers," Journal of the Optical Society of America B, vol. 26, no. 12, pp. B1-B6, 2009.

[3] E. Yablonovitch, T. J. Gmitter, and K. M. Leung, "Photonic band structure: the face-centered-cubic case employing nonspherical atoms," Physical Review Letters, vol. 67, no. 17, pp. 2295-2298, 1991.

[4] J. C. Knight, J. Broeng, T. A. Birks, and P. S. J. Russell, "Photonic band gap guidance in optical fibers," Science, vol. 282, no. 5393, pp. 1476-1478, 1998.

[5] Y. Fink, J. N. Winn, S. Fan et al., "A dielectric omnidirectional reflector," Science, vol. 282, no. 5394, pp. 1679-1682, 1998.

[6] M. J. Bloemer and M. Scalora, "Transmissive properties of Ag/ MgF2 photonic band gaps," Applied Physics Letters, vol. 72, no. 14, pp. $1676-1678,1998$.

[7] J. A. Monsoriu, R. A. Depine, M. L. Martínez-Ricci, and E. Silvestre, "Interaction between non-Bragg band gaps in ID metamaterial photonic crystals," Optics Express, vol. 14, no. 26, pp. 12958-12967, 2006.

[8] A. Suarez-Garcia, R. del Coso, R. Serna, J. Soils, and C. N. Afonso, "Controlling the transmission at the surface plasmon resonance of nanocomposite films using photonic structures," Applied Physics Letters, vol. 83, no. 9, pp. 1842-1844, 2003.

[9] J. H. Shahbazian and A. S. Karakashian, "LInear electrooptical effect on DPS-DNG layered structure," International Journal of Modern Physics B, vol. 23, no. 15, pp. 3205-3211, 2009.

[10] M. C. Larciprete, C. Sibilia, S. Paoloni, M. Bertolotti, F. Sarto, and M. Scalora, "Accessing the optical limiting properties of metallo-dielectric photonic band gap structures," Journal of Applied Physics, vol. 93, no. 9, pp. 5013-5017, 2003.

[11] L. M. Blinov, "Electro-optical effects in liquid crystals," Soviet Physics Uspekhi, vol. 17, article 658, 1975.

[12] H. Kato, T. Matsushita, A. Takayama, M. Egawa, K. Nishimura, and M. Inoue, "Theoretical analysis of optical and magnetooptical properties of one-dimensional magnetophotonic crystals," Journal of Applied Physics, vol. 93, no. 7, pp. 3906-3911, 2003.

[13] J. H. Shahbazian and A. S. Karakashian, "Magneto-optical effect of DPS-DNG layered structure," Journal of the Optical Society of America B, vol. 26, no. 12, pp. 2342-2346, 2009.

[14] Yu. Pisarevski, G. Tregubov, and Yu. Shaldin, "The electrooptical properties of $\mathrm{NH}_{4} \mathrm{H}_{2} \mathrm{PO}_{4} \mathrm{KH}_{2} \mathrm{PO}_{4}$, and $\mathrm{N}_{4}\left(\mathrm{CH}_{2}\right)_{6}$ crystals in UHF fields," Soviet Physics, Solid State, vol. 7, pp. 530531, 1965.

[15] R. Adhav, "Linear electro-optical effects in tetragonal phosphates and arsenates," Journal of the Optical Society of America A, vol. 59, no. 4, pp. 414-418, 1969. 

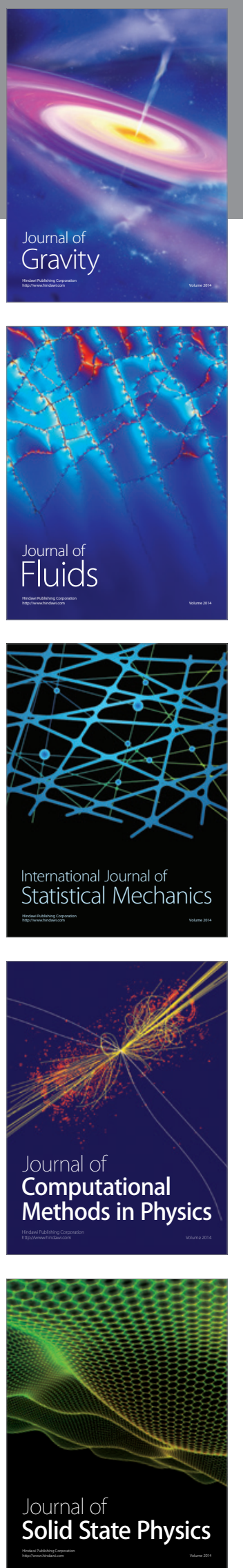
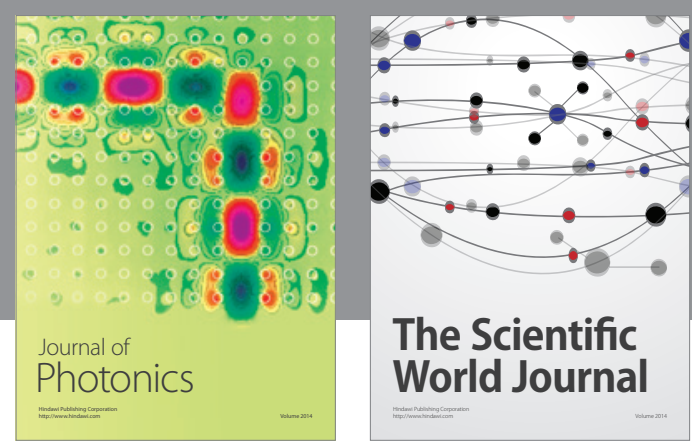

The Scientific World Journal

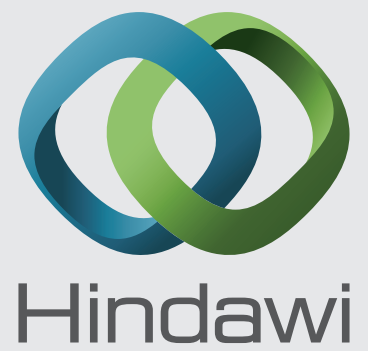

Submit your manuscripts at http://www.hindawi.com
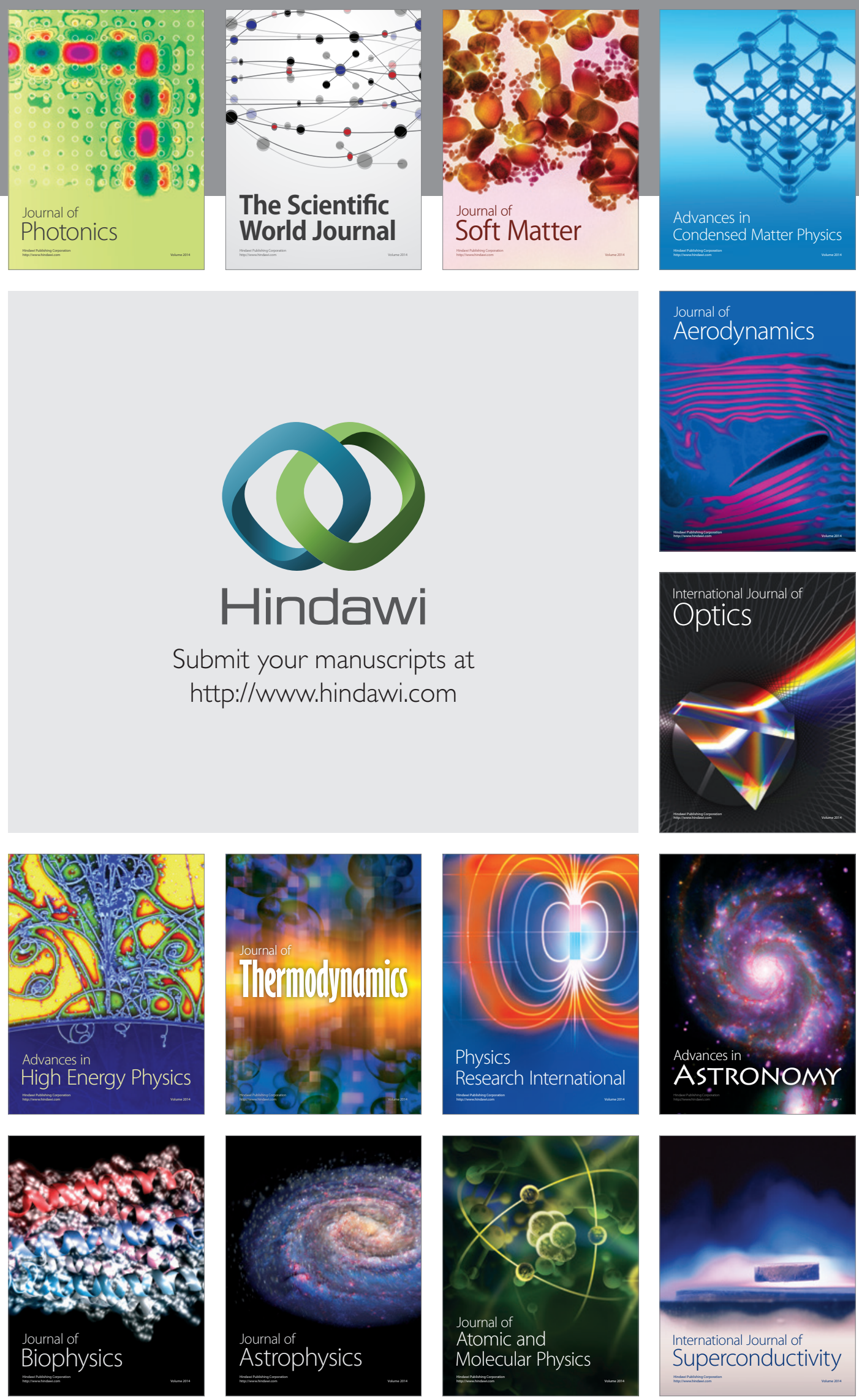
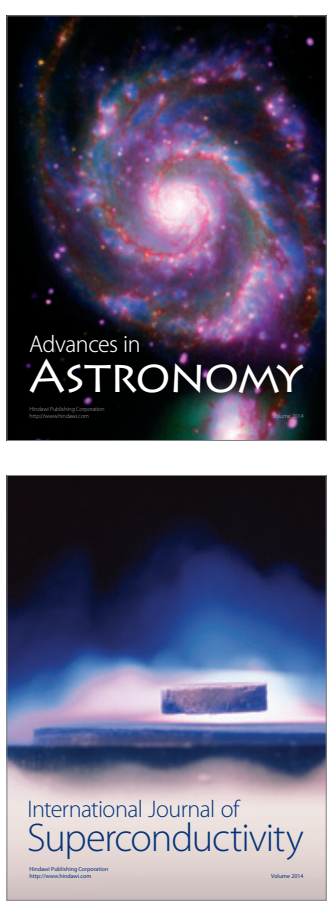